\title{
A Study of Fast Bunch Rotation in the Negative Mass Region
}

\author{
G. Rumolo, SL Accelerator Physics, CERN, Geneva, Switzerland \\ I. Hofmann, High Current Beam Physics, GSI, Darmstadt, Germany
}

\begin{abstract}
Fast bunch rotation of high-intensity proton or ion bunches above transition is - in principle - supported by the self-bunching effect of the attractive space charge force ("negative mass instability"). Due to the broad-band nature of the space charge impedance, the highest harmonics of this negative mass mode grow fast and inhibit compression, unless the bunch rotation is accelerated by a sufficiently high rf-voltage.

Using particle-in-cell simulation we establish the threshold below which effective compression is still possible. We find that the required rf-voltage for compression of a given bunch above transition can be reduced at most by a factor 2 compared with compression below transition, where space charge requires extra voltage.
\end{abstract}

\section{INTRODUCTION}

In a previous paper [1] we have shown that the rf requirements for compression of high-intensity proton (or ion) bunches cannot easily be reduced by approaching transition from below due to the dominating repulsive space charge forces. The attractiveness of small and negative slip factors is in fact limited, if space charge dominates the rf voltage and leads to a significant coherent momentum spread prior to completion of the compression. The question is thus of interest whether one could still gain rf-power by trying to carry out a fast bunch compression above transition where space charge is attractive and enhances the applied rf-voltage. However, the self-focusing effect of space charge is not limited to the envelope motion, but also applies to all higher order modes which is the well-known negative mass instability [2]. Since the growth rate increases with the mode number $n$ (up to some value $n^{*}$ ), the instability on the shortest wave-lengths is expected to appear first, which possibly limits or quenches the compression. In this paper we study the possibility of successful compression by using a sufficiently fast active rotation such as to overcome the destabilising effect of space charge. The plan of the paper is as follows. In Sect. II we review some basic concepts and analytical formulae concerning the negative mass instability. In Sect. III we discuss the simulation program and the effective space charge impedance resulting from solving Poisson's equation numerically. In section IV we present simulation results with conclusions in section V.

\section{REMARKS ON THE NEGATIVE MASS INSTABILITY}

Above transition perturbations of arbitrary wave-length in the beam current profile grow exponentially in time, because an energy variation induced by space charge on single particles results in a change of their angular velocity in the opposite direction. For wavelengths below a threshold (depending on the momentum spread of the beam), phase mixing due to particles of different longitudinal momenta prevents collective motion and stability is preserved. The mechanism that keeps the beam stable in this case is not quite the same as Landau damping [3]. In fact, this damping is not a kinetic process which involves energy transfer from the electrostatic wave to single particles resonant with the wave. The momentum spread here acts as a hydrodynamic property of the beam, regardless of the detailed shape of the distribution function, and assures stability above a certain mode number $n^{*}$. This is also confirmed by the fact that pure space charge waves are stationary in the beam frame, and distribution functions $f(p)$ usually have a zero slope at $p=0$, whereas Landau damping requires a running wave and a finite slope of the distribution function to ensure energy transfer to the wave from resonant particles. Stability boundary and curves at constant growth rate of instability in the impedance plane can be rigorously applied only to coasting beams. Nevertheless, the extension to bunched beams is generally accepted if the wave-length is small compared with the bunch length and the transit time of the perturbation over the bunch length is long compared with the growth time [4]. This "local Keil-Schnell" or "Boussard" criterion is certainly met in the present case, since negative mass modes always develop on very short wave-lengths and are stationary waves in the bunch frame. The rise time of an instability can be estimated analytically for a working point on the imaginary axis far outside the stability boundary by ignoring the momentum spread:

$$
\Delta \omega_{\text {inst }}= \pm \omega_{0}\left[\frac{\mathrm{e}|\eta| n \lambda_{\max }}{2 \pi \mathrm{m}_{0} \gamma \beta \mathrm{c}}\left|\Im\left(Z_{\|}(n)\right)\right|\right]^{1 / 2} .
$$

\section{COMPUTER SIMULATION}

The issue of fast bunch compression above transition has been studied by means of computer simulation using the code PATRIC. This is a particle-in-cell program, which solves the equations of motion in 3D, whereas Poisson's equation is solved in cylindrical $(r-z)$ geometry assum- 
ing a perfectly conducting beam pipe [5]. Using the parameters that are listed in Tab. 1, we have carried out a number of simulations of bunch rotation in order to explore the deteriorating effect of space charge above transition. Runs have been made with $10^{6}$ macroparticles on a $r-z$ grid of $32 \times 2048$ cells.

Table 1: Overview on the simulation parameters.

\begin{tabular}{|l|l|}
\hline Circumference, $2 \pi r_{0}$ & $20 \mathrm{~m}$ \\
Protons per bunch, $N_{b}$ & $10^{13}$ \\
$E_{\text {kin }}$ & $2 \mathrm{GeV}$ \\
Bunch length, $z_{i n}$ & $5 \mathrm{~m}$ \\
Momentum spread $\left(\delta p / p_{0}\right)_{i n}$ & 0.002 \\
Beam radius, $R_{b}$ & $0.02 \mathrm{~m}$ \\
Pipe radius, $R_{p}$ & $0.1 \mathrm{~m}$ \\
$\gamma_{t}$ & 2.56 \\
Compression factor, $\chi$ & 0.2 \\
\hline
\end{tabular}

\subsection{Space charge impedance}

In order to get a correct modelling of the negative mass instability up to highest frequencies, it is essential to compare the spectrum of the space charge impedance seen by the simulation beam with the theoretical expectation. While $\left|Z_{\| s c}(n)\right|$ increases linearly with $n$ for low harmonic numbers, a more refined model is needed for large $n$. Bisognano [6] obtained a Lorentzian shape for $\left|Z_{|| s c}(n)\right| / n$, which defines a cut-off at high mode numbers, but its application is limited to ultra-relativistic beams and to ratios $R_{p} / R_{b}$ of about 2. A general expression for the space charge impedance at all harmonic numbers and arbitrary $\gamma$ and $R_{p} / R_{b}$ has recently been obtained by AlKhateeb et al. [7]. In Fig.1 the curves that are compared with a numerical evaluation from PATRIC (dots) are the Bisognano formula (red dashed curve) and the newly corrected formula [7] (blue curve). It appears that the agreement between Al-Khateeb's formula for the space charge impedance and the numerically evaluated one is excellent, which confirms that the corrected expression for the space charge impedance coming from Ref. [7] can be used for the analytical estimates in this study.

\subsection{Instability rise times}

From the diagram in Fig. 1 and by using the instability maps in the impedance plane for the bunch of Table 1, we can estimate the harmonic number where the negative mass instability is expected to appear first, and its corresponding growth time.

The instability rise time can be plotted as a function of the harmonic number, as is shown in Fig. 2. The asymptote $n=650$ due to reaching the stability region comes from the equality:

$$
\frac{\Im\left[Z_{||}(650)\right]}{650}=2 \pi F \frac{\beta \mathrm{m}_{0} \mathrm{c} \gamma|\eta|}{\mathrm{e} \lambda_{\max }}\left(\frac{\delta p}{p_{0}}\right)^{2}=17.63 \Omega,
$$

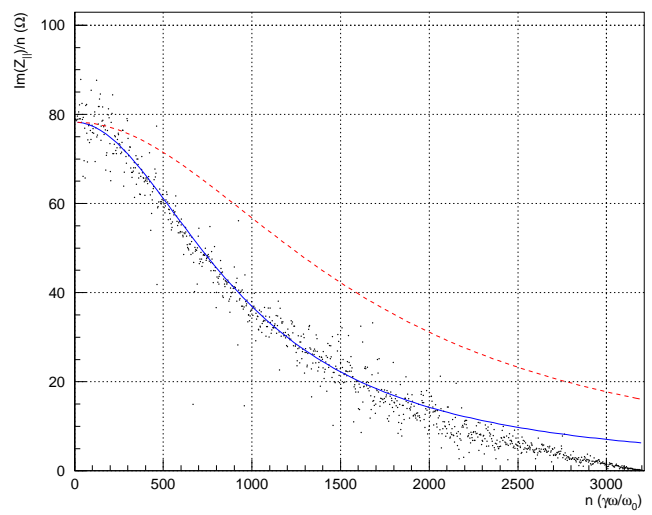

Figure 1: Space charge impedance seen by the beam.

whereas the minimum rise time occurs for harmonic numbers around 250 .

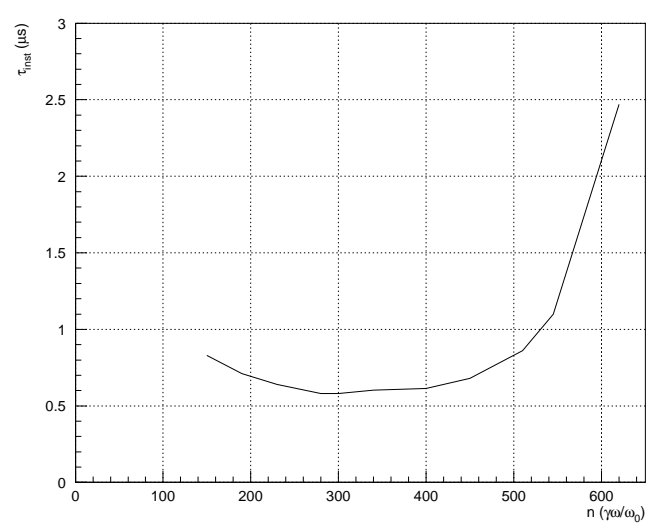

Figure 2: Rise times versus harmonic number for the bunch of Table I.

\section{COMPRESSION SIMULATIONS}

It is straightforward to derive that, having defined the $\Sigma$ parameter as [1]:

$$
\Sigma=-\frac{3 g_{0} N_{b} \mathrm{r}_{p}}{\beta^{2} \gamma^{3} \eta z_{b}\left(\delta p / p_{0}\right)_{b}^{2}},
$$

the condition for a successful bunch compression above transition energy without concerns relative to negative mass instability reads:

$$
|\Sigma| \leq 1.4
$$

This translates into a condition on the compression ratio $\chi$ which is $\chi \geq 2.5$. As the compression ratio cannot by definition exceed unity, this means that it is never possible to get a bunch compression only using the attractive space charge force above transition. This should not be surprising, because being all negative mass modes suppressed when the impedance working point is inside the stability region, the mode $n=1$ is equally suppressed 
and no self-compression can therefore take place. It is of interest to investigate whether conditions exist, in which an active bunch compression can be successfully carried out even for larger values of $|\Sigma|$, when a large fraction of the negative mass modes are also excited. The advantage would obviously be to save rf power, because the voltage required by a compression at a higher $|\Sigma|$ once the compression factor has been fixed can be sensibly smaller.

Following the general parameters of Table I, and modifying one of them at a time, we have simulated the evolution of a proton bunch in order to show how it can be rotated above transition using an appropriate voltage, even when space charge is not negligible and the negative mass effect on high harmonic numbers would otherwise make the process inefficient. The voltage that is tentatively applied to achieve compression is the one which would be required for compression of the same proton bunch having the same $|\eta|$, but below transition and neglecting space charge. This voltage is exactly twice smaller than the one which should be applied below transition in order to carry out the compression against space charge [1].
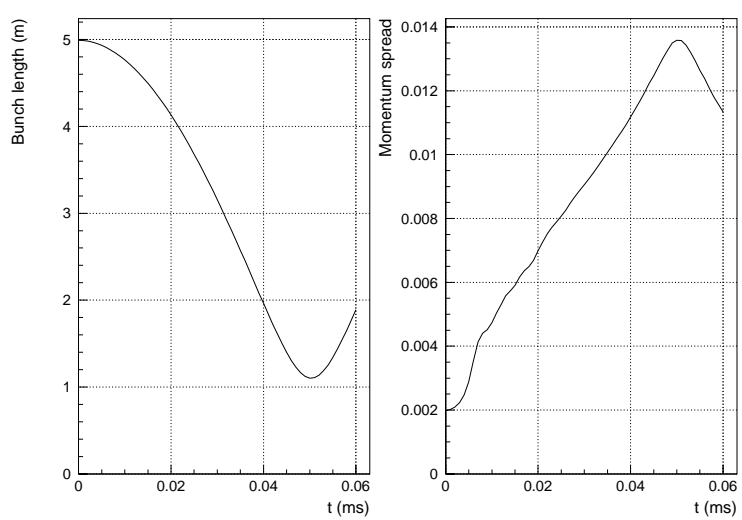

Figure 3: Evolution of bunch length and momentum spread during compression of a bunch having $\Sigma=-6$.

We start from simulating exactly the case of Table I. Fig. 3 shows how momentum spread and bunch length evolve during the process. The compression appears to be still effective, and the final bunch is about $1 \mathrm{~m}$ long with small tails. The over-momentum observed at the end of the compression is only about $17 \%$ higher than the expected value. The parameters of Table I correspond to a beam which has $\Sigma=-6$. By carrying out more simulations in which the beam energy and the machine geometry were kept constant, we have found out that every variation of the other parameters that still delivers an equal value of $\Sigma$ does not affect the success of the compression. The bunch compression becomes uneffective if we try to operate it at a higher value of $\Sigma$, as appears from Fig. $4(\Sigma=-12)$. In reality, the $|\Sigma|$ criterion applies only when the energy of the beam and all geometric parameters are fixed. Otherwise, the ratio between the compression time (as calculated
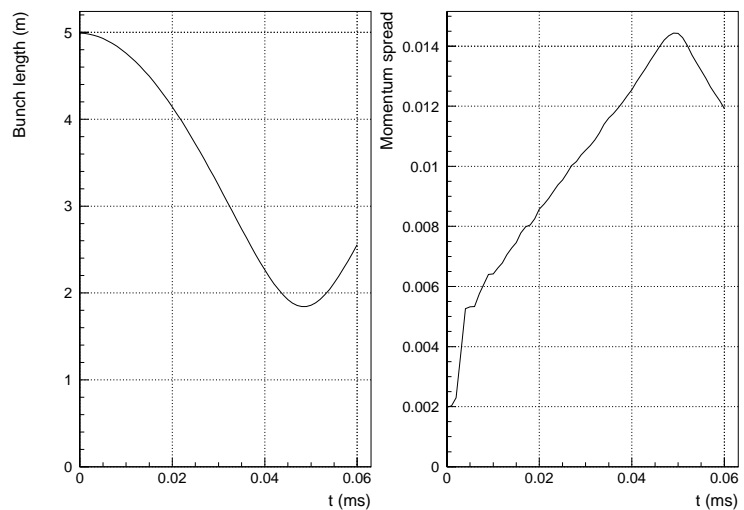

Figure 4: Evolutioh of bunch length and momentum spread during compression of a bunch having $\Sigma=-12$.

ignoring space charge) and the rise time of the fastest instability (evaluated following the procedure explained in the previous section), $T_{\text {compr }} / \tau_{\text {inst }}$ should be kept lower than about 100 .

\section{CONCLUSIONS}

In this paper we have shown that it is possible to carry out the bunch rotation of an intense proton beam by using an adequate voltage, which in some conditions can be up to twice smaller than the same required below transition at the same value of $|\eta|$. The voltage used for the rotation is in fact exactly the one needed below transition for the same value of $|\eta|$, but having ignored space charge. The general criterion to establish whether the bunch can be compressed by this voltage must consist in making sure that the ratio between compression time and fastest instability rise time, $T_{\text {compr }} / \tau_{\text {inst }}$, is maintained below a threshold of about 100 .

\section{REFERENCES}

[1] G. Franchetti, I. Hofmann and G. Rumolo, Phys. Rev. ST Accel. Beams 3, 084201 (2000).

[2] P. J. Channell LBL PEP Note 104, p. 28 (1974)

[3] A. Hofmann, in Proceedings of the fifth advanced Accelerator Physics Course, Greece, 1993, edited by S. Turner (CERN-95-06) p. 275

[4] A. G. Ruggiero and V. G. Vaccaro, CERN Report No. CERN-ISR-TH/68-33, 1968 (unpublished)

[5] I. Hofmann and G. Kalisch, Phys. Rev. E 53, 2807 (1996).

[6] Joseph J. Bisognano, in Proceedings of the Fifth European Particle Accelerator Conference (EPAC96), Edit. S. Myers, A. Pacheco, R. Pascual, Ch. Petit-Jean-Genaz and J. Poole, Institute of Physics Publishing, Bristol and Philadelphia, Vol. I, p. 328 (1996).

[7] A. Al-Khateeb, O. Boine-Frankenheim, I. Hofmann, and G. Rumolo, Phys. Rev. E 63, 026503 (2001).

[8] M. Reiser, Theory and Design of Charged Particle Beams, John Wiley \& Sons, Inc. 1994. 